\title{
Challenges and Opportunities in Materials Science with Next Generation Monochromated EELS
}

\author{
P. A. Crozier ${ }^{1}$, J. Zhu ${ }^{1,2}$, T. Aoki ${ }^{2}$, P. Rez $^{3}$, W. J. Bowman ${ }^{1}$, R.W. Carpenter ${ }^{2}$, O.L. Krivanek ${ }^{4}$, N. \\ Dellby $^{4}$, T.C. Lovejoy ${ }^{4}$, R.F. Egerton ${ }^{5}$ \\ ${ }^{1}$ School for Engineering of Matter, Transport and Energy, Arizona State University, Tempe, AZ 85287 , \\ USA \\ ${ }^{2}$ LeRoy Erying Center for Solid State Science, Arizona State University, Tempe, AZ 85287, USA \\ ${ }^{3}$ Department of Physics, ASU, Tempe, AZ 85287, USA \\ ${ }^{4}$ Nion Co., 1102 8th St, Kirkland, WA 98033, USA \\ ${ }^{5}$ Department of Physics, University of Alberta, Edmonton T6G 2E1, Canada
}

The recent development of monochromated scanning transmission electron microscopes (STEM) offering energy resolutions of better than $20 \mathrm{meV}$ and electron probes of $1 \AA$ in size provides a completely new tool to materials characterization. Unique opportunities opened by access to ultra-high energy resolution low loss EELS include determination of optical properties in the IR, bandgap mapping, detection of defect interband states and localized vibrational spectroscopy. While techniques such as Raman and IR spectroscopy have been routinely used to characterize phonon modes in solids for many years, the vibrational excitations probed with focused fast electron beams is largely unexplored. At ASU we are currently applying ultra-high energy resolution low-loss EELS to a variety of materials that are important in fields such as energy, environmental science and information technology. Here we show representative initial results on carbons, oxides and metal hydrides. All data were acquired on a newly installed Nion UltraSTEM equipped with a probe corrector and monochromator [1].

The optical properties of carbonaceous atmospheric aerosols are an important contributor to radiative forcing for climate change. By applying Kramers-Kronig techniques to energy-loss spectra acquired from the Nion, it is now possible to determine optical properties well into the IR. Figure 1 shows the low-loss spectrum recorded from two types of carbonaceous particles. The high energy resolution and smaller zero-loss tails allow the refractive index to be accurately determined out to photon wavelengths of $2500 \mathrm{~nm}$, thus essentially cover most of the incoming solar spectrum [2].

Local measurement of bandgaps and states within the gap is of great importance for opto-electronic materials. Figure 2 shows the low-loss spectra from ceria $\left(\mathrm{CeO}_{2}\right)$ and a ceria co-doped with $\mathrm{Gd}$ and $\mathrm{Pr}$ $\left(\mathrm{Ce}_{0.85} \mathrm{Gd}_{0.11} \mathrm{Pr}_{0.04} \mathrm{O}_{2-\delta}\right)$. From EELS, local bandgaps were about $3.5 \mathrm{eV}$ and in some regions additional peaks were detected within the bandgap (Figure 2b). Interestingly, all the ceria based samples showed significant uniform intensity within the bandgap which is absent in spectra recorded from BN under identical conditions. The origin of the gap intensity in the oxides will be discussed in terms of Cerenkov radiation, defects, surface layers, and sample preparation.

At lower energy transfers, localized phonon spectroscopy becomes possible. We have been able to identify vibrational peaks in a variety of compounds like $\mathrm{SiO}_{2}$ which match the Raman spectrum [1]. The behavior and interpretation of some of these very low-loss features is not yet well understood. Figure 3 shows two regions of the low-loss spectrum from TiH2. The peak at $150 \mathrm{meV}$ is prominent under aloof beam conditions but is less easy to see when the beam is placed on the sample. When the 
primary electron beam passes directly through the sample, the $150 \mathrm{meV}$ peak may be more difficult to detect due to the larger electronic signal or because of rapid loss of $\mathrm{H}_{2}$.

Ultrahigh energy resolution EELS is an exciting new tool for characterization of materials at high spatial resolution. However, to realize its full potential, considerable experimental and theoretical work must be undertaken to develop a fundamental understanding of this form of EELS.

\section{References}

[1] O.L. Krivanek et al, these proceedings (2014)

[2] J. Zhu et al, these proceedings (2014)

[3] The authors acknowledge support of the NIST 60NANB10D022, NSF Graduate Research Fellowship Program (DGE-1311230), NSF DMR 1308085, NSF MRI-R2 959905 and DOE DESC0004954. The authors acknowledge the use of facilities in the John M. Cowley Center for High Resolution Microscopy at Arizona State University.
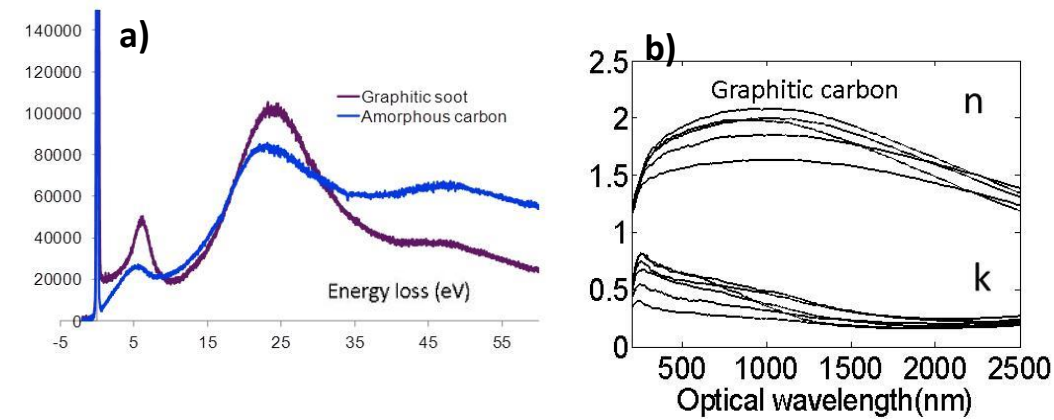

Figure 1: (a) EELS from two forms of carbonaceous particles.

(b) Complex refractive index derived from EELS covering photon wavelength range 200$2500 \mathrm{~nm}$.
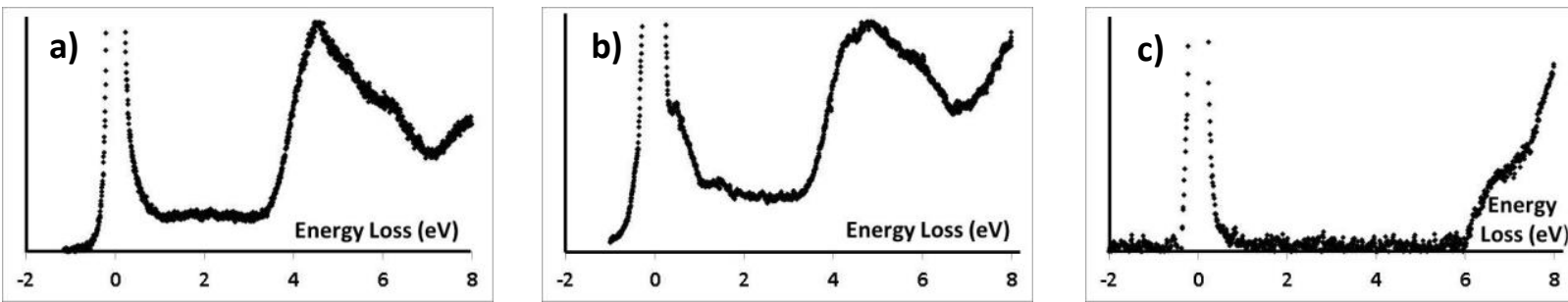

Figure 2: Low-loss spectra from a) $\mathrm{CeO} 2$, b) $\mathrm{Ce} 0.85 \mathrm{Gd} 0.11 \operatorname{Pr} 0.04 \mathrm{O} 2-\delta$ and c) hexagonal $\mathrm{BN}$.
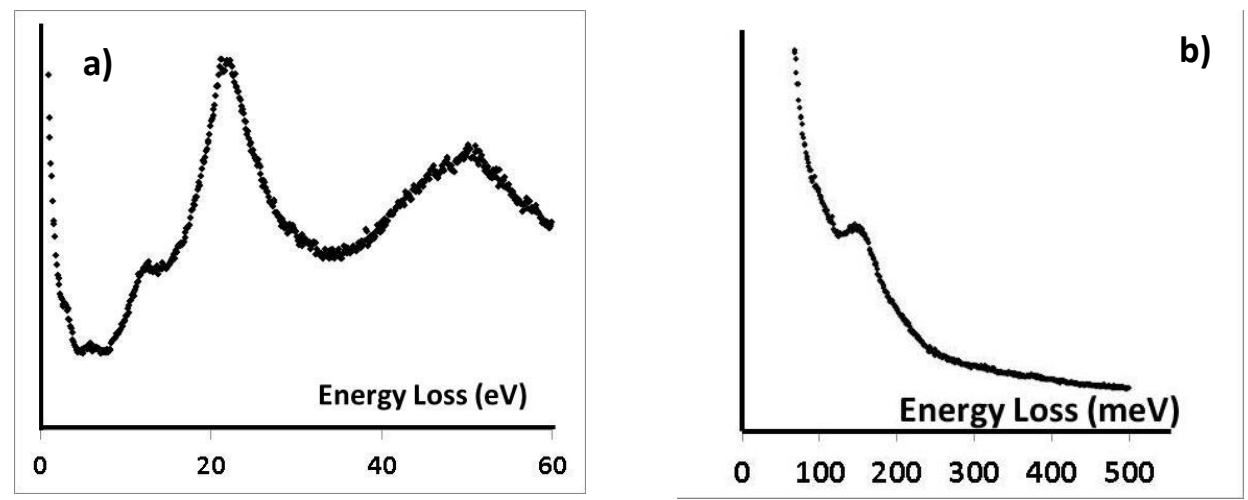

Figure 3: EELS from $\mathrm{TiH} 2$ showing a) wide energy range on sample and b) very low energy-loss region in aloof beam mode ( $\sim 5 \mathrm{~nm}$ off sample). 